\title{
Novel Function of Sprouty4 as a Regulator of Stemness and Differentiation of Embryonic Stem Cells
}

\author{
Jae-Young Lee ${ }^{1}$, Sunghyun Park ${ }^{1}$, Kwang-Soo Kim ${ }^{1}$, Jeong-Jae Ko ${ }^{1}$, Soohong Lee ${ }^{1}$, \\ ${ }^{\dagger}$ Keun Pil Kim ${ }^{2}$ and ${ }^{\dagger}$ Kyung-Soon Park ${ }^{1}$ \\ ${ }^{1}$ Dept. of Biomedical Science, College of Life Science, CHA University, Seoul 06135, Korea \\ ${ }^{2}$ Dept. of Life Science, Chung-Ang University, Seoul 06975, Korea
}

\begin{abstract}
Sprouty (Spry) genes encode inhibitors of the receptor tyrosine kinase signaling cascade, which plays important roles in stem cells. However, the role of Spry4 in the stemness of embryonic stem cells has not been fully elucidated. Here, we used mouse embryonic stem cells (mESCs) as a model system to investigate the role of Spry4 in the stem cells. Suppression of Spry4 expression results in the decreases of cell proliferation, EB formation and stemness marker expression, suggesting that Spry4 activity is associated with stemness of mESCs. Teratoma assay showed that the cartilage maturation was facilitated in Spry4 knocked down mESCs. Our results suggest that Spry4 is an important regulator of the stemness and differentiation of mESCs.
\end{abstract}

Key words : Sprouty4, Embryonic stem cell, Differentiation

\section{INTRODUCTION}

Sprouty (Spry) was first discovered as a common antagonist of fibroblast growth factor (FGF) and epidermal growth factor (EGF) signaling pathways in Drosophila (Hacohen et al., 1998; Kramer et al., 1999). In a search of the Expressed Sequence Tag database, three human homologs of the fly gene, designated hSpryl-3, were identified (Hacohen et al., 1998). The fourth mammalian homolog, hSpry4, was later discovered in mice and humans (De Maximy et al., 1999; Leeksma et al., 2002). Emerging evidence shows that Spry specifically inhibits mitogen-activated protein kinase (MAPK) signaling activated by a wide range of ligands, including FGF, platelet-derived growth factor (Gross et al., 2001), vascular endothelial growth factor (Impagnatiello et al., 2001), nerve growth factor (Wong et al., 2002), brainderived neurotrophic factor (Gross et al., 2007), and glial cell line-derived neurotrophic factor (Ishida et al., 2007), but does not affect MAPK signaling activated by EGF (Sasaki et al., 2001). Spry2 reportedly potentiates biological effects induced by EGF via inhibiting EGF receptor degradation (Wong et al., 2001; Egan et al., 2002; Rubin et al., 2003). These findings suggest that Spry isoforms regulate MAPK signaling in a negative or positive manner, possibly depending on the subcellular context.

Autocrine FGF-induced extracellular signal-regulated kinase

\footnotetext{
Manuscript received 2016, Received in revised form 2016, Accepted 2016

${ }^{\dagger}$ Corresponding Author : Kyung-Soon Park, Dept. of Biomedical Science, College of Life Science, CHA University, Seoul 06135, Korea. Tel. : +82-31881-7144, E-mail : kspark@cha.ac.kr, Keun Pil Kim, Dept. of Life Science, Chung-Ang University, Seoul 06975, Korea. Tel. : +82-2-820-5792, E-mail: kpkim@cau.ac.kr

This is an Open Access article distributed under the terms of the Creative Commons Attribution Non-Commercial License (http:// creativecommons.org/licenses/by-nc/3.0) which permits unrestricted non-commercial use, distribution, and reproduction in any medium, provided the original work is properly cited.
} 
(ERK) $1 / 2$ signaling occurs in embryonic stem cells (ESCs) and performs controversial functions to maintain the pluripotency of mouse ESCs (mESCs). It primes mESCs to initiate their differentiationin response to external differentiationinducing stimuli as well as inducing the expression of inhibitors of FGF signaling to generate a negative-feedback loop that would facilitate reversion back to the original unprimed state (Kunath et al., 2007; Stavridis et al., 2007; Stavridis et al., 2010). ERK signaling can determine whether mesenchymal stem cells commit to the osteogenic orchondrogenic lineage. Inhibition of the ERK1/2 pathway completely abolishes chondrogenic differentiation and augments osteogenic commitment (Pelaez et al., 2012). Spry2 and Spry4 are the most highly expressed Spry isoforms in human ESCs. Knockdown of Spry2 decreases the proliferation and increases the death of human ESCs, whereas knockdown of Spry4 enhances the survival of the cells. This indicates that the Spry family regulates pathways involved in the proliferation and survival of ESCs (Felfly and Klein, 2013).

Although ERK signaling is a core signaling pathway to sustain the pluripotency of mESCs and to regulate the lineage commitment of mesenchymal stem cells, it is unknown whether Spry4 sustains the differentiation potential of mESCs and adult stem cells functions as it does in human ESCs. Here, we used mESCs as models to investigate the role of Spry4 in the stem cells. We report that Spry4 contributes to the maintenance of stemness and balanced differentiation of mESCs. When Spry4 was suppressed, stemness of mESCs was disrupted and the development of cartilage was facilitated interatomas formed by mESCs. Our results reveal that Spry4 is an important regulator of the stemness and differentiation of mESCs.

\section{MATERIALS AND METHODS}

\section{Reagents and cell culture}

J1 mESCs (cat \#SCRC-1010) were purchased from ATCC (www.atcc.org) and maintained as described previously (Jung et al., 2012). To for membryoidbodies (EBs), mESCs were trypsinized to form a single-cell suspension and subsequently cultured on uncoated Petri dishes in ESC medium lacking leukemia inhibitory factor (LIF).

\section{Genetic modification of mESCs}

shRNA plasmids targeting mouse Spry4 were purchased (TL509780, Origene, MD, USA) to generate a stable Spry4knockdown cell line. mESCs were grown to $80 \%$ confluence in 24-well plates, then transfected with shRNA plasmids using Lipofectamine 2000 (Invitrogen) following the manufacturer's instructions. Stable mESCs expressing shRNA for Spry4 were selected in $1 \mu \mathrm{g} / \mathrm{mL}$ of puromycincontaining culture medium. Knockdown of Spry4 expression was confirmed with real-time reverse transcription polymerase chain reaction (RT-PCR) analysis and immunoblot analysis.

\section{RNA extraction and real-time RT-PCR}

Total RNA was extracted using TRIzol (Invitrogen), and 2-5 $\mu \mathrm{g}$ of total RNA was reverse-transcribed using the SuperScriptII TM First-Strand Synthesis System (Invitrogen) according to the manufacturer's instructions. Real-time RTPCR was performed using cDNA with the Quantitect SYBR Green PCR Kit (Qiagen, CA, USA). Reactions were performed in triplicate using an Exicycler ${ }^{\mathrm{TM}} 96$ real-time quantitative thermal block (Bioneer). For quantification, target genes were normalized against the glyceraldehyde 3-phosphate dehydrogenase gene.

\section{Immunoblotting}

Whole-cell extracts were prepared, and 20-50 $\mu$ g of protein was resolved by SDS-PAGE and blotted using antibodies against Spry4 (ab59785, Abcam, MA, USA), phosphoStat3 (Tyr-705; \#9131, Cell Signaling Technology, Beverly, MA, USA), Stat3 (sc-482, Santa Cruz, CA, USA), cMyc 
(ab32072, Abcam), Nanog (ab80892, Abcam), Sox2 (sc20088, Santa Cruz), phospho-ERK (M9692, Sigma), ERK2 (sc-154, Santa Cruz), and $\beta$-actin (sc-47778, Santa Cruz). Immunoreactivity was detected by enhanced chemiluminescence (Amersham, Buckinghamshire, England).

\section{Teratoma formation}

For the teratoma formation assay, cells were trypsinized, and $5 \times 10^{5}$ cells were suspended in a DMEM/Matrigel solution (BD Biosciences Inc., Franklin Lakes, NJ, USA) (1:1 ratio $(\mathrm{v} / \mathrm{v}))$. The cell/Matrigel suspension was injected subcutaneously into NOD/SCID mice (Charles River Laboratories, Yokohama, Japan). Teratoma formation was examined for 6 weeks after injection. The experiments were reviewed and approved by the Institutional Animal Care and Use Committee of CHA University. All procedures were performed in accordance with the Guidelines for the Care and Use of Laboratory Animals published by the US National Institutes of Health (NIH publication no. 85-23, revised 1996).

\section{Statistical analysis}

Graphical data are presented as means $\pm \mathrm{SD}$. Each experiment was performed at least three times and subjected to statistical analysis. Statistical significance between two groups was determined using Student's $t$-test. A $p$ value below 0.05 was considered significant. Statistical analysis was performed using the SAS statistical package v.9.13 (SAS Inc., Cary, NC).

\section{RESULTS AND DISCUSSION}

Activation of the FGF-ERK signaling pathway is required for naïve mESCs to initiate lineage differentiation by exiting their self-renewal status. Therefore, FGF signaling should be tightly regulated at multiple levels in ESCs. We previously reported that Spry1, a member of the Spry family of proteins, is necessary to balance the self-renewal and pluripotency of
mESCs by inhibiting ERK1/2 signaling under self-renewal culture conditions (Jung et al., 2012). To increase our understanding of the regulatory mechanisms of ERK1/2 signaling in mESCs, we further investigated the role of Spry4 in mESCs.

We first examined whether Spry4 modulates phosphorylation of ERK1/2 in mESCs. When Spry4 expression was suppressed by transfection of shRNA-targeting Spry4, ERK phosphorylation was significantly increased, suggesting that Spry4 suppresses ERK1/2 signalingin mESCs (Fig. 1A). We next engineered mESCs expressing green fluorescent protein and Spry4-targeting shRNA to generate a stable Spry4-knockdown mESC line (Spry4 KD), and then examined the effect of Spry4 suppression on the morphology and proliferation of these cells. Although Spry4 KD showed strong phosphorylation of ERK, it maintained the undifferentiated round shape of $\mathrm{mESCs}$ and alkaline phosphatase activity (Fig. 1B, 1C). However, the proliferation of Spry4 $\mathrm{KD}$ was lower than that of control cells (Fig. 1D). To examine whether altered Spry4 expression affects mESC self-renewal, we examined the expression of undifferentiated ESC markers. Expression of Oct4 and Sox 2 was decreased at the mRNA and protein levels, whereas expression of c-Myc, Nanog, and phosphorylated Stat3 was not affected by suppression of Spry4 expression (Fig. 1E). We examined the ability of Spry4 KD to maintain an undifferentiated state by analyzing the efficiency of secondary EB formation (Felfly \& Klein, 2013). The ability of Spry4 KD to maintain an undifferentiated state was com-parable to that of control $\mathrm{mESCs}$, indicating that alteration of Spry4 did not affect the self-renewal of mESCs, despite the finding that suppression of Spry4 decreased cell pro-liferation and reduced expression of Sox2 and Oct4 (Fig. 1F). Our results are consistent with previous reports that Fgf4-null ESCs behave normally in the undifferentiated state in the presence of LIF, although they cannot exit the pluripotent state and initiate differentiation when LIF is removed (Kunath et al., 2007). 
A

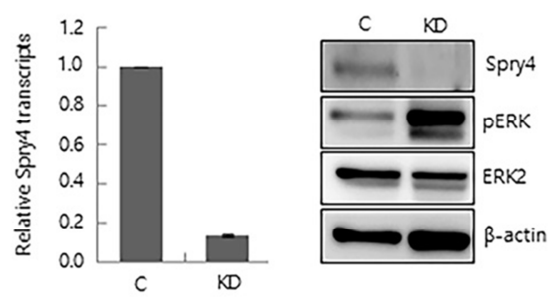

$\mathrm{E}$

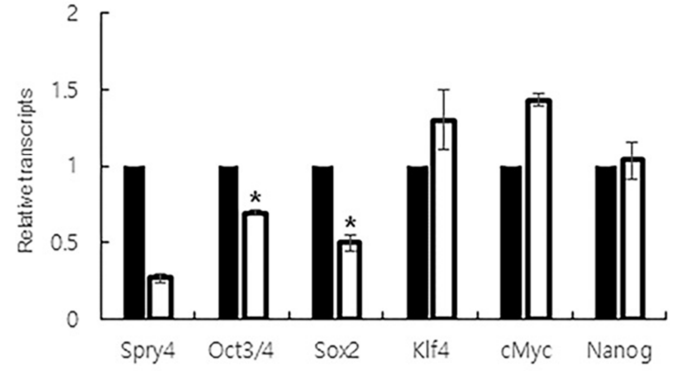

B

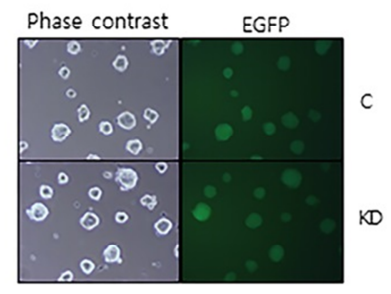

$\mathrm{C}$

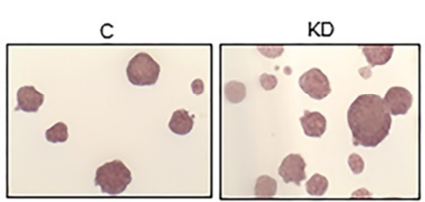

D

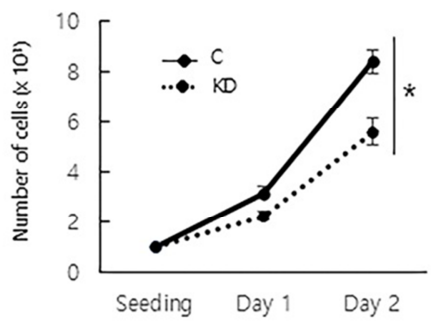

$\mathrm{F}$

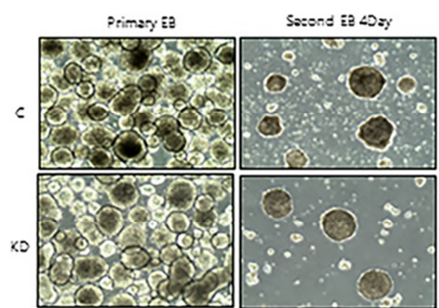

Fig. 1. The effect of Spry4 suppression on the self-renewal of mESCs. (A) Expression of Spry4 in Spry4-knockdown mESCs (KD) was quantitatively analyzed by real-time RT-PCR (left) and immunoblotting (right). The effect of Spry4 suppression on the level of phospho-ERK was analyzed by immunoblotting (right). (B) The morphologies of Spry4 KD and control mESCs were observed by phase contrast microscopy and fluorescence microscopy. (C) The growth of Spry4 KD and control mESCs were compared by counting cells at $24 \mathrm{~h}$ (Day 1) and $48 \mathrm{~h}$ (Day 2) after seeding. (D) Expression of self-renewal genes was analyzed by real-time RT-PCR (left) and immunoblotting (right). (E) Alkaline phosphatase staining of Spry4 KD and control mESCs. (F) Primary and secondary EB formation abilities of Spry $4 \mathrm{KD}$ and control mESCs. All values are means \pm standard deviation of at least three independent experiments. ${ }^{*} P<0.05$ based on the student's $t$-test. Abbreviations: $\mathrm{C}, \mathrm{mESCs}$ with control shRNA integration.

Autocrine FGF/ERK signaling is a pro-differentiation signal (Kunath et al., 2007); therefore, we hypothesized that the differentiation potential of Spry4 KD was higher than that of control mESCs. To test this hypothesis, we analyzed the impact of Spry4 suppression on the differrentiation of mESCs in xenografts. As expected, Spry4KD gave rise to larger teratomas than control mESCs (Fig. 2A). We next analyzed differentiation of the three germ lineages interatomas by hematoxylin-eosin (H\&E) staining. The pro- portions of mesoderm tissues, including cartilage, bone, neuroglia and primitive mesoderm, as well as endoderm tissues, such as glands, were significantly increased in 6week-old teratomas formed by Spry4KD (Fig. 2B, 2C). On the other hand, the proportion of immature cartilage was significantly lower in teratomas formed by Spry4 KD than in control teratomas, suggesting that the balanced differrentiation potential of $\mathrm{mESCs}$ was disturbed by suppression of Spry4. The expression level of Spry4 is much lower than 
A.

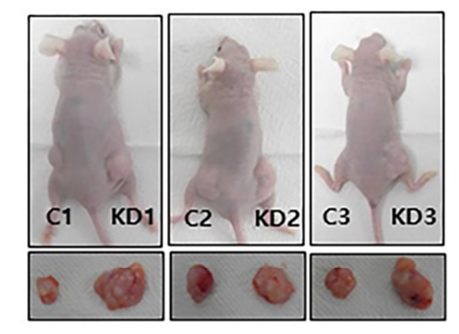

B.

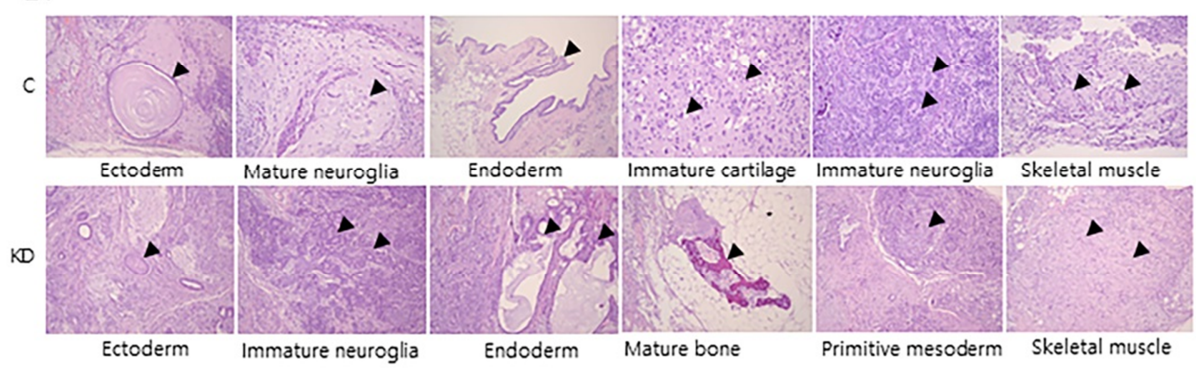

C.

\begin{tabular}{|c|c|c|c|c|c|c|c|}
\hline & differentiated tissue (\%) & KD1 & $\mathrm{KD} 2$ & KD3 & $\mathrm{C} 1$ & $\mathrm{C} 2$ & $\mathrm{C} 3$ \\
\hline size $(\mathrm{mm})$ & & $14 \star 7$ & $12 \star 7$ & $14 * 5$ & $9 * 4$ & $9.5 * 7$ & $5 \star 1$ \\
\hline Ectoderm & squamous epithelial cell & 2 & 1 & 0 & 0 & 0 & 1 \\
\hline \multirow[t]{8}{*}{ Mesoderm } & cartilage, mature & 14 & 11 & 15 & 0 & 0 & 0 \\
\hline & cartilage, immature & 0 & 0 & 1 & 79 & 18 & 37 \\
\hline & bone, mature & 11 & 4 & 5 & 0 & 0 & 0 \\
\hline & skeletal muscle, immature & 2 & 4 & 1 & 0 & 1 & 0 \\
\hline & fat, mature & 0 & 0 & 0 & 0 & 0 & 0 \\
\hline & neuroglia, mature & 31 & 20 & 18 & 10 & 35 & 20 \\
\hline & neuroglia, immature & 20 & 25 & 20 & 10 & 45 & 40 \\
\hline & primitive mesodermal stroma & 10 & 20 & 30 & 0 & 0 & 0 \\
\hline Endoderm & mucinous and non-mucinous glands & 10 & 15 & 10 & 1 & 1 & 2 \\
\hline total & & 100 & 100 & 100 & 100 & 100 & 100 \\
\hline
\end{tabular}

Fig. 2. Teratoma formation and differentiation of the three germ layers by Spry4 KD. (A) Spry4KD (KD) and control mESCs were injected into NOD/SCID mice and teratoma development was examined. The size of teratomas was examined 6 weeks after injection. (B) H\&E staining and data analysis of teratoma sections were performed by apathologist. Mature or immature germ layer differentiationis indicated by arrows. (C) Table of the relative percentages of differentiated tissues in teratomas formed by Spry4 KD and control mESCs. Abbreviations: C, mESCs with control shRNA integration.

that of Spry1 in mESCs (Jung et al., 1012). Despite it slow expression level, our results show that Spry4 plays a major role in control of the differentiation ability of mESCs.

In our analysis of the lineage differentiation of teratomas formed by mESCs, we noticed that mature cartilage was highly developed in teratomas formed by Spry4 KD in comparison to control teratomas (Fig. 2C). Cartilage loss is a typical degenerative disease because cartilage has a limited regeneration potential; however, effective therapy remains elusive. Therefore, further work to investigate the detailed mechanism by which Spry4 enhances cartilage differentiation will contribute to enhance the differentiation efficiency of stem cells.

\section{ACKNOWLEDGEMENTS}

This work was performed with the support of the Cooperative Research Program for Agriculture Science \& Technology Development (PJ010033), Rural Development Administration, Republic of Korea. This work was also supported by the Korea Science and Engineering Foundation (KOSEF) of the Korean government (MOST) (2012M3A9 C6050367).

\section{REFERENCES}

Barna M, Niswander L (2007) Visualization of cartilage 
formation: Insight into cellular properties of skeletal progenitors and chondrodysplasia syndromes. Dev Cell 12:931-941.

Cabrita MA, Christofori G (2008) Sprouty proteins, masterminds of receptor tyrosine kinase signaling. Angiogenesis 11:53-62.

Caron MM, Emans PJ, Coolsen MM, Voss L, Surtel DA, Cremers A, van Rhijn LW, Welting TJ (2012) Redifferentiation of dedifferentiated human articular chondrocytes: Comparison of 2D and 3D cultures. Osteoarthritis Cartilage 20:1170-1178.

Chan RJ, Johnson SA, Li Y, Yoder MC, Feng GS (2003) A definitive role of Shp-2 tyrosine phosphatase in mediating embryonic stem cell differentiation and hematopoiesis. Blood 102:2074-2080.

de Maximy AA, Nakatake Y, Moncada S, Itoh N, Thiery JP, Bellusci S (1999) Cloning and expression pattern of a mouse homologue of drosophila sprouty in the mouse embryo. Mech Dev 81:213-216.

Egan JE, Hall AB, Yatsula BA, Bar-Sagi D (2002) The bimodal regulation of epidermal growth factor signaling by human Sprouty proteins. Proc Natl Acad Sci U S A 99:6041-6046.

Felfly H, Klein OD (2013) Sprouty genes regulate proliferation and survival of human embryonic stem cells. Sci Rep 3:2277.

Gross I, Armant O, Benosman S, de Aguilar JL, Freund JN, Kedinger M, Licht JD, Gaiddon C, Loeffler JP (2007) Sprouty2 inhibits BDNF-induced signaling and modulates neuronal differentiation and survival. Cell Death Differ 14:1802-1812.

Gross I, Bassit B, Benezra M, Licht JD (2001) Mammalian sprouty proteins inhibit cell growth and differentiation by preventing ras activation. J Biol Chem 276:4646046468.

Hacohen N, Kramer S, Sutherland D, Hiromi Y, Krasnow MA (1998) sprouty encodes a novel antagonist of FGF signaling that patterns apical branching of the Drosophila airways. Cell 92:253-263.

Haigl B, Mayer CE, Siegwart G, Sutterluty H (2010) Sprouty4 levels are increased under hypoxic conditions by enhanced mRNA stability and transcription. Biol Chem 391:813-821.

Horowitz A, Simons M (2008) Branching morphogenesis. Circ Res 103:784-795.

Impagnatiello MA, Weitzer S, Gannon G, Compagni A, Cotten M, Christofori G (2001) Mammalian sprouty-1 and -2 are membrane-anchored phosphoprotein inhibitors of growth factor signaling in endothelial cells. J Cell Biol 152:1087-1098.

Ishida M, Ichihara M, Mii S, Jijiwa M, Asai N, Enomoto A, Kato T, Majima A, Ping J, Murakumo Y, Takahashi M (2007) Sprouty2 regulates growth and differentiation of human neuroblastoma cells through RET tyrosine kinase. Cancer Sci 98:815-821.

Jung JE, Moon SH, Kim DK, Choi C, Song J, Park KS (2012) Sprouty1 regulates neural and endothelial differrentiation of mouse embryonic stem cells. Stem Cells Dev 21:554-561.

Kramer S, Okabe M, Hacohen N, Krasnow MA, Hiromi Y (1999) Sprouty: A common antagonist of FGF and EGF signaling pathways in Drosophila. Development 126: 2515-2525.

Kunath T, Saba-El-Leil MK, Almousailleakh M, Wray J, Meloche S, Smith A (2007) FGF stimulation of the Erk1/2 signalling cascade triggers transition of pluripotent embryonic stem cells from self-renewal to lineage commitment. Development 134:2895-2902.

Lee SH, Schloss DJ, Jarvis L, Krasnow MA, Swain JL (2001) Inhibition of angiogenesis by a mouse sprouty protein. J Biol Chem 276:4128-4133.

Leeksma OC, Van Achterberg TA, Tsumura Y, Toshima J, Eldering E, Kroes WG, Mellink C, Spaargaren M, Mizuno K, Pannekoek H, de Vries CJ (2002) Human 
sprouty 4, a new ras antagonist on $5 \mathrm{q} 31$, interacts with the dual specificity kinase TESK1. Eur J Biochem 269: 2546-2556.

Li J, Zhao Z, Liu J, Huang N, Long D, Wang J, Li X, Liu Y (2010) MEK/ERK and p38 MAPK regulate chondrogenesis of rat bone marrow mesenchymal stem cells through delicate interaction with TGF-beta1/Smads pathway. Cell Prolif 43:333-343.

Rubin C, Litvak V, Medvedovsky H, Zwang Y, Lev S, Yarden Y (2003) Sprouty fine-tunes EGF signaling through interlinked positive and negative feedback loops. Curr Biol 13:297-307.

Sasaki A, Taketomi T, Wakioka T, Kato R, Yoshimura A (2001) Identification of a dominant negative mutant of Sprouty that potentiates fibroblast growth factor- but not epidermal growth factor-induced ERK activation. J Biol Chem 276:36804-36808.

Schonwasser DC, Marais RM, Marshall CJ, Parker PJ (1998) Activation of the mitogen-activated protein kinase/ extracellular signal-regulated kinase pathway by conventional, novel, and atypical protein kinase $\mathrm{C}$ isotypes. Mol Cell Biol 18:790-798.

Stavridis MP, Collins BJ, Storey KG (2010) Retinoic acid orchestrates fibroblast growth factor signalling to drive embryonic stem cell differentiation. Development 137: 881-890.

Stavridis MP, Lunn JS, Collins BJ, Storey KG (2007) A discrete period of FGF-induced Erk1/2 signalling is required for vertebrate neural specification. Development 134:2889-2894.

Taniguchi K, Ishizaki T, Ayada T, Sugiyama Y, Wakabayashi Y, Sekiya T, Nakagawa R, Yoshimura A (2009a) Sprouty4 deficiency potentiates Ras-independent angiogenic signals and tumor growth. Cancer Sci 100:1648-1654.

Taniguchi K, Sasaki K, Watari K, Yasukawa H, Imaizumi T, Ayada T, Okamoto F, Ishizaki T, Kato R, Kohno R, Kimura H, Sato Y, Ono M, Yonemitsu Y, Yoshimura A (2009b) Suppression of Sproutys has a therapeutic effect for a mouse model of ischemia by enhancing angiogenesis. PLoS One 4:e5467.

Warburton D (2008) Developmental biology: order in the lung. Nature 453:733-735.

Wong ES, Fong CW, Lim J, Yusoff P, Low BC, Langdon WY, Guy GR (2002) Sprouty2 attenuates epidermal growth factor receptor ubiquitylation and endocytosis, and consequently enhances Ras/ERK signalling. EMBO J 21:4796-4808.

Wong ES, Lim J, Low BC, Chen Q, Guy GR (2001) Evidence for direct interaction between Sprouty and Cbl. J Biol Chem 276:5866-5875.

Yoon HH, Bhang SH, Shin JY, Shin J, Kim BS (2012) Enhanced cartilage formation via three-dimensional cell engineering of human adipose-derived stem cells. Tissue Eng Part A 18:1949-1956. 